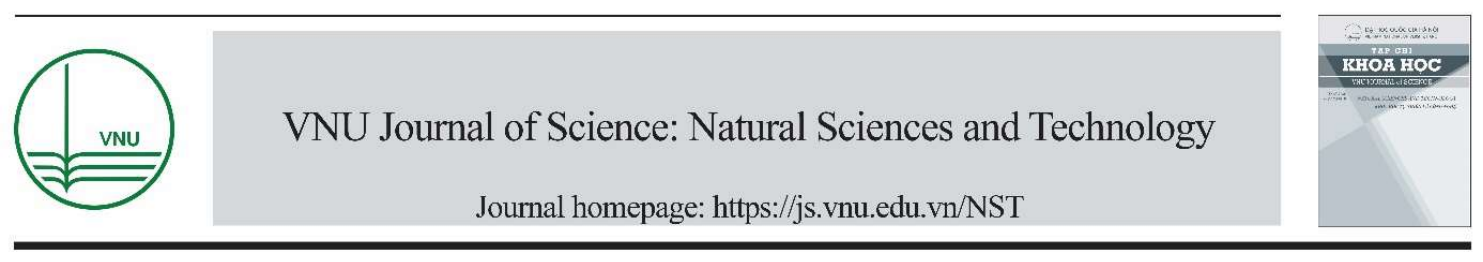

\title{
Original Article \\ Influence of Antioxidants on Physio-Chemical Properties of the Mixture of Rice Bran Oil and Rapeseed Oil for Transformers
}

\author{
Nguyen Van Dung*, Pham Thanh Liem, Trinh Quoc Anh \\ Cantho University, $3 / 2$ street, Cantho, Vietnam \\ Received 9 October 2020 \\ Revised 19 February 2021; Accepted 28 June 2021
}

\begin{abstract}
This study evaluated the effect of antioxidants on the physical and chemical parameters of a mixture of rice oil and rape-seed oil for using as insulating transformer oil. Antioxidants such as butylated hydroxytoluene, tertiary butyl hydroquinone and propyl gallate were used. Experimental results showed that these three additives increased the oxidation stability index of the oil mixture, but tertiary butyl hydroquinone had the greatest effect and increased the oxidation stability index by 4 times. However, tertiary butyl hydroquinone insignificantly changes the physical and chemical parameters of the oil mixture except for the breakdown strength. This parameter of the oil mixture was increased by about $24 \%$ when tertiary butyl hydroquinone $(0,3 \mathrm{wt} \%)$ was added. In addition, it was observed that tertiary butyl hydroquinone inhibited the aging process of the oil mixture at high temperatures. With the presence of tertiary butyl hydroquinone, the viscosity of the aged oil mixture only increased by $16 \%$ instead of up to $36 \%$ in case of without addition of the antioxidant under without oxygen or low concentration of oxygen.
\end{abstract}

Keywords: Rice oil, Rape-seed Oil, Transformers, Ageing, Antioxidants.

${ }^{*}$ Corresponding author.

Email address: nvdung@ctu.edu.vn

https://doi.org/10.25073/2588-1140/vnunst.5139 


\title{
Ảnh hưởng của chất chống oxy hóa đến đặc tính vật lý và hóa học của hỗn hợp dầu cám và dầu hạt cải dùng cho máy biến áp
}

\author{
Nguyễn Văn Dũng*, Phạm Thanh Liêm, Trịnh Quốc Anh \\ Trường Đại học Cần Thơ, Đường 3/2, Cần Thơ, Việt Nam \\ Nhận ngày 9 tháng 10 năm 2020 \\ Chỉnh sửa ngày 19 tháng 02 năm 2021; Chấp nhận đăng ngày 28 tháng 6 năm 2021
}

\begin{abstract}
Tóm tắt: Nghiên cứu này đánh giá ảnh hưởng của chất chống oxy hóa đến các thông số vật lý và hóa học của hỗn hợp dầu cám và dầu hạt cải để sử dụng làm dầu cách điện cho máy biến áp phân phối. Các chất chống oxy hóa như dibutylhydrotoluen (hoặc 2,6-di-tecbutyl-4-metylphenol), tecbutylhydroquinon (hoặc 2-tec-butylbenzen-1,4-diol) và propyl gallat đã được sử dụng. Kết quả thí nghiệm cho thấy các chất phụ gia này đã làm tăng độ ổn định oxy hóa của hỗn hợp dầu nhưng tecbutylhydroquinon có ảnh hưởng lớn nhất và đã tăng độ ổn định oxy hóa gấp 4 lần. Tuy nhiên tecbutylhydroquinon không làm thay đổi đáng kể các thông số vật lý và hóa học của hỗn hợp dầu ngoại trừ độ bền điện. Thông số này của hỗn hợp dầu đã tăng khoảng $24 \%$ khi được bổ sung tecbutylhydroquinon $(0,3 \mathrm{wt} \%)$. Ngoài ra, tec-butylhydroquinon còn cho thấy khả năng hạn chế sự lão hóa hỗn hợp dầu ở nhiệt độ cao. Kết quả là với sự hiện diện của tec-butylhydroquinon, độ nhớt của hỗn hợp dầu sau lão hóa chỉ tăng $16 \%$ thay vì tăng đển 36\% trong trường hợp không có chất chống oxy hóa khi thí nghiệm trong điều kiện không có oxy hoặc nồng độ oxy thấp.
\end{abstract}

Tù̀ khóa: Dầu cám, dầu hạt cải, máy biến áp, sự lão hóa, chất chống oxy hóa.

\section{1. Đặt vấn đề}

Dầu cách điện có nguồn gốc thực vật đã được ứng dụng thành công trong máy biến áp phân phối và ngày càng trở nên phổ biến [1]. Điều này là do dầu thực vật có các ưu điểm như độ bền điện cao, điểm chớp cháy cao, phân hủy hoàn toàn và không độc đối với con người và môi trường [2,3]. Một số loại dầu cách điện được chế tạo từ dầu hướng dương, dầu đậu nành, dầu bắp và dầu hạt cải đã được thương mại hóa trên thị trường [2,3]. Một số loại dầu khác như dầu cám, dầu đậu phộng, dầu dừa...đang được nghiên cứu [4-6]. Trong các loại dầu này, dầu cám có tiềm năng lớn nhất do đạt hầu hết các chỉ tiêu về kỹ thuật cũng như có giá thành tương đương hoặc thấp hơn so với các loại dầu còn lại [6]. Tuy nhiên dầu cám có hai nhược điểm là có nhiệt độ đông đặc cao và dễ bị oxy hóa đặc biệt là dưới tác động của nhiệt độ cao $[4,5]$. Do đó nghiên cứu này sẽ trình bày phương pháp hạ thấp điểm đông đặc và nâng cao khả năng chống oxy hóa của dầu cám bằng cách hòa trộn với dầu hạt cải và sử dụng các chất chống oxy hóa như butylated hydroxytoluene (BHT), tec-butylhydroquinon (hoặc 2-tec-butylbenzen-1,4-diol) và propyl gallate $(\mathrm{PG})$. Các kết quả nghiên cứu trước đây cho thấy $B H T$ và $T B H Q$ đã có hiệu quả cao đối với dầu thực vật [5]. Ngoài ra, PG cũng đã nâng cao khả năng chống oxy hóa của nhiên liệu sinh học [7]. Vì vậy, nghiên cứu này sẽ khảo sát ảnh hưởng của $B H T$, TBHQ và $P G$ đến các thông số vật lý và hóa học của hỗn hợp dầu cám và dầu hạt cải.

\footnotetext{
*Tác giả liên hệ.

Địa chỉ email: nvdung@ctu.edu.vn

https://doi.org/10.25073/2588-1140/vnunst.5139
} 


\section{Bố trí thí nghiệm và phương pháp thí nghiệm}

\subsection{Bố trí thí nghiệm và phương pháp thí nghiệm}

Thí nghiệm xác định độ ổn định oxy hóa của dầu được thực hiện theo phương pháp rancimat phù hợp với tiêu chuẩn $\mathrm{EN} 14112$ như mô tả ở Hình 1. Thí nghiệm được thực hiện tại $110^{\circ} \mathrm{C}$ với lưu lượng không khí là $10 \mathrm{~L} / \mathrm{h}$. Khi dầu bị oxy dưới tác động của nhiệt độ cao, các axit có phân tử lượng thấp dễ bay hơi hình thành. Các axit này được dẫn đến cốc đựng nước cất. Do đó, độ dẫn điện của nước trong cốc sẽ tăng theo thời gian thí nghiệm và độ ổn định oxy hóa được xác định khi độ dẫn điện của nước tăng đột ngột.

Thí nghiệm xác định điện áp đánh thủng của các mẫu dầu được bố trí như Hình 2 . Thí nghiệm được thực hiện với điện áp AC-50 Hz theo tiêu chuẩn IEC 60156 với khe hở điện cực là $2,5 \mathrm{~mm}$.

Thí nghiệm lão hóa dầu được thực hiện theo tiêu chuẩn IEC 61125C. Dầu được lão hóa tại $120^{\circ} \mathrm{C}$ trong $164 \mathrm{~h}$ có bơm không khí với lưu lượng là $0,15 \mathrm{~L} / \mathrm{h}$ và không có bơm không khí.

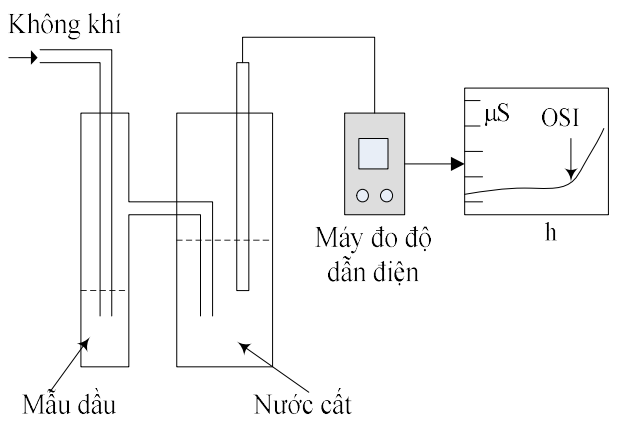

Hình 1. Bố trí thí nghiệm đo độ ổn định oxy hóa [8].

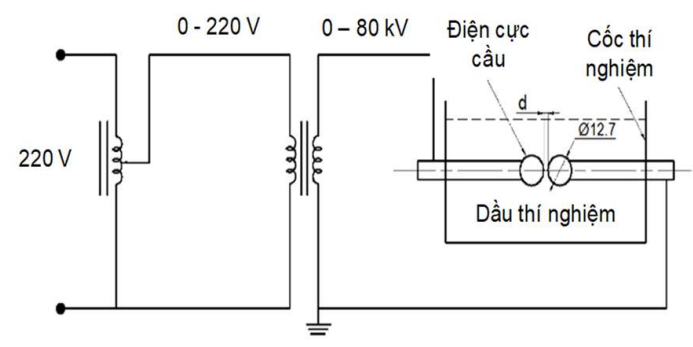

Hình 2. Bố trí thí nghiệm đo điện áp đánh thủng.

\subsection{Mẫu dầu và chất chống oxy hóa}

Dầu cám và dầu hạt cải tinh luyện từ công ty SIMPLY được sử dụng để thực hiện các thí nghiệm. Thành phần hóa học của hai loại dầu này được trình bày ở Bảng 1 .

Bảng 1 . Thành phần hóa học của dầu cám và dầu hạt cải tinh chế (wt\%)

\begin{tabular}{|c|l|c|c|}
\hline TT & \multicolumn{1}{|c|}{ Thành phần } & $\begin{array}{c}\text { Dầu cám } \\
{[10]}\end{array}$ & $\begin{array}{c}\text { Dầu hạt cải } \\
{[11]}\end{array}$ \\
\hline 1 & Axit béo bão hòa & 20,1 & 5,78 \\
\hline 2 & $\begin{array}{l}\text { Axit béo không } \\
\text { bão hòa đơn }\end{array}$ & 42,2 & 62,7 \\
\hline 3 & $\begin{array}{l}\text { Axit béo không } \\
\text { bão hòa đa }\end{array}$ & 36,5 & 28,5 \\
\hline 4 & Khác & 1,2 & 3,02 \\
\hline
\end{tabular}

Bảng 2. Thông số kỹ thuật của dầu

\begin{tabular}{|c|c|c|c|c|}
\hline TT & Chỉ tiêu & $\begin{array}{l}\text { Dầu } \\
\text { cám } \\
{[12]}\end{array}$ & $\begin{array}{l}\text { Dầu hạt } \\
\text { cải [12] }\end{array}$ & $\begin{array}{l}\text { ASTM } \\
\text { D6871 }\end{array}$ \\
\hline 1 & $\begin{array}{l}\text { Độ nhớt tại } 40^{\circ} \mathrm{C} \\
\text { (cSt) }\end{array}$ & 36,9 & 35,2 & $\leq 50$ \\
\hline 2 & $\begin{array}{l}\text { Điểm đông đặc } \\
\left({ }^{\circ} \mathrm{C}\right)\end{array}$ & 0 & -21 & $\leq-10$ \\
\hline 3 & $\begin{array}{l}\text { Điểm chớp cháy } \\
\left({ }^{\circ} \mathrm{C}\right)\end{array}$ & 328 & 342 & $\geq 275$ \\
\hline 4 & $\begin{array}{l}\text { Khối lượng riêng } \\
\text { tại } 40^{\circ} \mathrm{C}(\mathrm{g} / \mathrm{ml})\end{array}$ & 0,917 & 0,92 & $\leq 0,96$ \\
\hline 5 & $\begin{array}{l}\text { Hàm lượng nước } \\
(\mathrm{mg} / \mathrm{kg})\end{array}$ & 106,1 & 95,1 & $\leq 200$ \\
\hline 6 & $\begin{array}{l}\text { Điện áp đánh } \\
\text { thủng tại khe hở } \\
2,5 \mathrm{~mm}(\mathrm{kV})\end{array}$ & 49,5 & 41 & $\geq 30$ \\
\hline 7 & $\begin{array}{l}\text { Hệ số tồn hao } \\
\text { điện môi tại } 25^{\circ} \mathrm{C} \\
(\%)\end{array}$ & 0,12 & 0,14 & $\leq 0,2$ \\
\hline 8 & $\begin{array}{l}\text { Hàm lượng lưu } \\
\text { huỳnh ăn mòn }\end{array}$ & $\begin{array}{l}\text { Không } \\
\text { có }\end{array}$ & $\begin{array}{l}\text { Không } \\
\text { có }\end{array}$ & $\begin{array}{l}\text { Không } \\
\text { có }\end{array}$ \\
\hline 9 & $\begin{array}{l}\text { Trị số axít (mg } \\
\mathrm{KOH} / \mathrm{g})\end{array}$ & $\leq 0,006$ & $\leq 0,01$ & $\leq 0,06$ \\
\hline
\end{tabular}

Do có tî̉ lệ phần trăm axit béo bão hòa cao hơn nên điểm đông đặc của dầu cám sẽ cao hơn dầu hạt cải [1]. Ngoài ra, tổng phần trăm của axit béo bão hòa và không bão hòa đơn không chênh lệch lớn giữa dầu cám và dầu hạt cải nên dự đoán độ ổn định oxy hóa sẽ tương tự nhau giữa hai loại 
dầu. Đặc tính hóa học, vật lý và điện của hai loại dầu này được cho ở Bảng 2 . Rõ ràng chỉ có điểm đông đặc của dầu cám không đạt yêu cầu. Trước khi tiến hành thí nghiệm, các mẫu dầu được sấy ở $85^{\circ} \mathrm{C}$ trong chân không (10 mbar) trong vòng $48 \mathrm{~h}$ để loại bỏ nước và khí hòa tan. Chất chống oxy hóa $\mathrm{BHT}$, TBHQ và $\mathrm{PG}$ được sử dụng với nồng độ là $0,3 \mathrm{wt} \%$ theo đề xuất của các nghiên cứu trước đây [9]. BHT và TBHQ được mua từ Sigma Aldrich trong khi $\mathrm{PG}$ được cung cấp từ HIMEDIA.

\section{Kết quả và thảo luận}

\section{1. Điểm đông đặc của hỗn hợp dầu}

Hình 3 cho thấy điểm đông đặc của hỗn hợp dầu giảm khi tỉ lệ dầu cám trong hỗn hợp giảm, và đạt $-12^{\circ} \mathrm{C}$ khi dầu cám chỉ chiếm khoảng $40 \%$ thể tích của hỗn hợp dầu. Kết quả này là do điểm đông đặc của dầu cám cao hơn so với dầu hạt cải $\left(0^{\circ} \mathrm{C}\right.$ so với $\left.-21^{\circ} \mathrm{C}\right)$. Như vậy, hỗn hợp dầu chứa $40 \%$ dầu cám và $60 \%$ dầu hạt cải có điểm đông đặc thấp hơn một ít so với giới hạn trên theo qui định tại tiêu chuẩn $\mathrm{ASTM}-\mathrm{D} 6871\left(\leq-10^{\circ} \mathrm{C}\right)$, và tỉ lệ này được chọn trong các thí nghiệm tiếp theo.

\section{2. Độ ổn định oxy hóa của hồn hợp dầu}

Độ ổn định oxy hóa (OSI) của các mẫu dầu được xác định bằng phương pháp rancimat và kết quả đo được trình bày ở Bảng 3 . Từ bảng này cho thấy, OSI của hỗn hợp dầu giảm so với giá trị của dầu cám $(1,4 \mathrm{~h}$ so với $2,2 \mathrm{~h})$. Kết quả này có thể là do dầu cám có chứa hàm lượng chất chống oxy hóa tự nhiên cao nên có OSI cao hơn so với dầu hạt cải $(2,2 \mathrm{~h}$ so với $1,1 \mathrm{~h})$ dẫn đến kết quả là nếu tỉ lệ dầu cám trong hốn hợp thấp thì OSI của hỗn hợp sẽ thấp. Ngoài ra, cũng có thể là do thành phần hóa học của dầu cám khó bị oxy hóa hơn so với dầu hạt cải. Các giả thuyết này cần được làm rõ trong các nghiên cứu tiếp theo. Khi bổ sung chất chống oxy hóa với nồng độ $0,3 \mathrm{wt} \%$, OSI của hỗn hợp dầu tăng lên đáng kể và đạt giá trị lớn nhất trong trường hợp của TBHQ $(6,0$ h). Kết quả này cho thấy hiệu quả của việc sử dụng TBHQ để nâng cao khả năng chống oxy hóa của hỗn hợp dầu cám và dầu hạt cải. Kết quả tương tự đã được trình bày trong các nghiên cứu trước đây $[5,13]$. Do đó TBHQ được chọn để tiếp tục thực hiện các thí nghiệm kế tiếp.

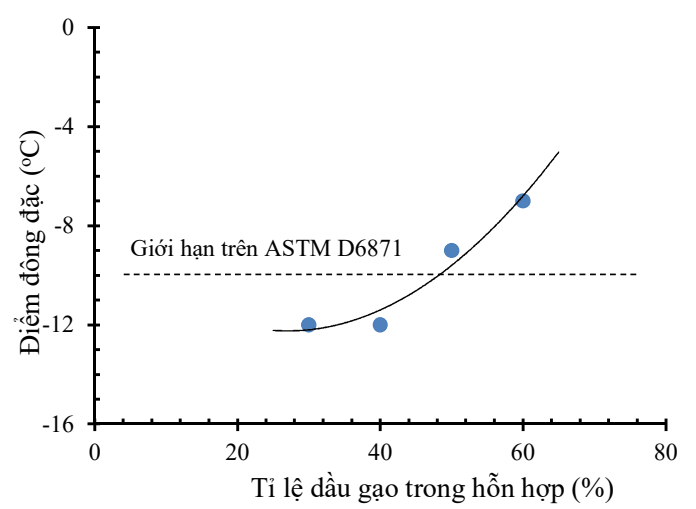

Hình 3. Tương quan giữa điểm đông đặc và tỉ lệ dầu cám trong hỗn hợp dầu.

Bảng 3. OSI của các mẫu dầu

\begin{tabular}{|c|l|c|}
\hline TT & \multicolumn{1}{|c|}{ Mẫu dầu } & OSI $(\mathrm{h})$ \\
\hline 1 & Dầu cám & $2,2 \pm 0,24$ \\
\hline 2 & Dầu hạt cải & $1,1 \pm 0,16$ \\
\hline 3 & Dầu cám+dầu hạt cải & $1,4 \pm 0,21$ \\
\hline 4 & Dầu cám+dầu hạt cải+BHT & $2,5 \pm 0,14$ \\
\hline 5 & Dầu cám+dầu hạt cải+TBHQ & $6,0 \pm 0,76$ \\
\hline 6 & Dầu cám+dầu hạt cải+PG & $2,9 \pm 0,21$ \\
\hline
\end{tabular}

\subsection{Sự lão hóa hỗn hợp dầu}

Hỗn hợp dầu được lão hóa theo tiêu chuẩn IEC $61125 \mathrm{C}$ với nồng độ TBHQ là 0,3 wt $\%$. Hỗn hợp dầu sau lão hóa được đo điện áp đánh thủng và độ nhớt. Kết quả thí nghiệm cho thấy sự lão hóa làm tăng điện áp đánh thủng của hỗn hợp dầu khoảng $27 \%$ đối với trường hợp không có TBHQ và $6 \%$ đối với trường hợp có TBHQ (Hình 4). Kết quả này là do hỗn hợp dầu khô hơn sau khi lão hóa. Kết quả tương tự thu được đối với dầu $\mathrm{FR}_{3}$ và Midel eN [14]. Ngoài ra, kết quả thí nghiệm còn cho thấy TBHQ đã tăng điện áp đánh thủng của hỗn hợp dầu khoảng $24 \%$ đối với trường hợp dầu mới và $3 \%$ đối với trường hợp sau lão hóa. Kết quả tương tự đã được trình bày bởi các tác giả khác $[15,16]$. Kết quả này là do chất chống oxy hóa đã gia tăng sự phân nhánh 
của dòng điện tích khi di chuyển trong dầu. Điều này sẽ làm giảm vận tốc dịch chuyển của dòng điện tích và nâng cao điện áp đánh thủng $[17$, $18]$.

Hình 5 trình bày ảnh hưởng của sự lão hóa đến độ nhớt của hỗn hợp dầu. Trong trường hợp có bơm không khí, độ nhớt của hỗn hợp dầu sau lão hóa tăng khoảng $53 \%$ đối với mẫu dầu không chứa $\mathrm{TBHQ}$ và $42 \%$ đối với mẫu dầu có chứa TBHQ. Kết quả này cho thấy khi chịu tác động của nhiệt độ cao và tiếp xúc với oxy trong không khí, dầu bị lão hóa nhanh chóng [19] và rõ ràng hiệu quả chống lão hóa của TBHQ không cao trong trường hợp này. Trong trường hợp không có bơm không khí, độ nhớt của hỗn hợp dầu sau lão hóa tăng khoảng $36 \%$ đối với mẫu dầu không có $\mathrm{TBHQ}$ và $16 \%$ đối với mẫu dầu có $\mathrm{TBHQ}$. Điều này chỉ ra rằng $\mathrm{TBHQ}$ có hiệu quả cao trong việc hạn chế sự lão hóa dầu trong điều kiện không có oxy hoặc tồn tại oxy với nồng độ thấp.

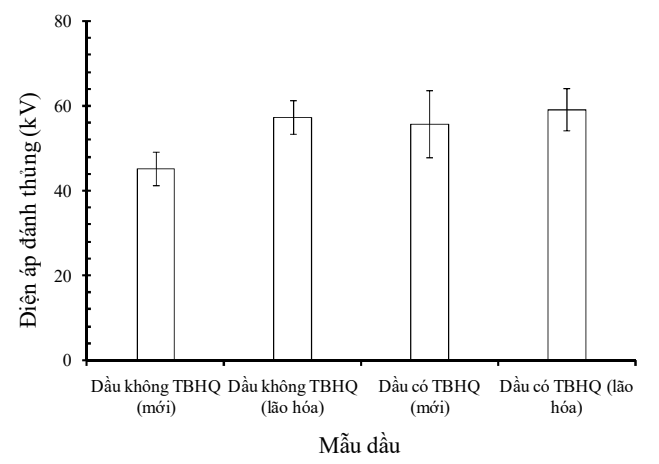

Hình 4. Ảnh hưởng của sự lão hóa đến điện áp đánh thủng của hỗn hợp dầu (có bơm không khí).

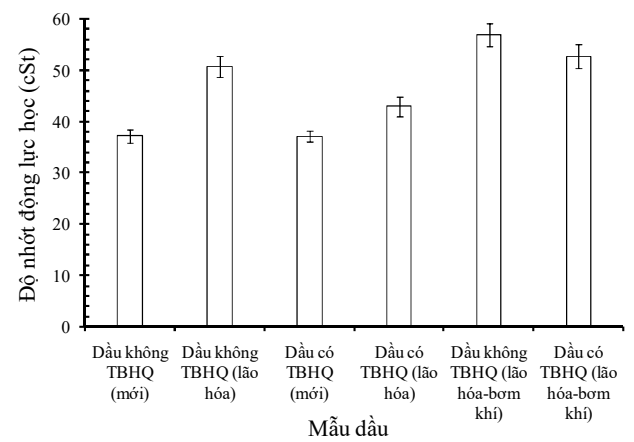

Hình 5. Ảnh hưởng của sự lão hóa đến độ nhớt của hỗn hợp dầu.

\subsection{Thông số kỹ thuật của hốn hợp dầu chưa $T B H Q$}

Bảng 4 thể hiện các thông số kỹ thuật của hỗn hợp dầu chứa TBHQ $(0,3 \mathrm{wt} \%)$. Các thông số dầu được xác định tại các phòng thí nghiệm thuộc Trường Đại học Cần Thơ, Trung tâm kỹ thuật tiêu chuẩn đo lường chất lượng Cần Thơ và Công ty thí nghiệm điện Miền Nam. Kết quả này cho thấy hỗn hợp dầu đạt tất cả các thông số kỹ thuật theo tiêu chuẩn ASTM D6871.

Bảng 4. Thông số kỹ thuật của hỗn hợp dầu

\begin{tabular}{|c|l|c|c|}
\hline TT & \multicolumn{1}{|c|}{ Chỉ tiêu } & $\begin{array}{c}\text { Hỗn hợp } \\
\text { dầu }\end{array}$ & $\begin{array}{c}\text { ASTM } \\
\text { D6871 }\end{array}$ \\
\hline 1 & $\begin{array}{l}\text { Độ nhớt tại } 40^{\circ} \mathrm{C} \\
(\mathrm{cSt})\end{array}$ & 36,1 & $\leq 50$ \\
\hline 2 & Điểm đông đặc $\left({ }^{\circ} \mathrm{C}\right)$ & -12 & $\leq-10$ \\
\hline 3 & $\begin{array}{l}\text { Điểm chớp cháy } \\
\left({ }^{\circ} \mathrm{C}\right)\end{array}$ & 335 & $\geq 275$ \\
\hline 4 & $\begin{array}{l}\text { Khối lượng riêng } \\
\left.\text { tại 40 }{ }^{\circ} \mathrm{C} / \mathrm{ml}\right)\end{array}$ & 0,92 & $\leq 0,96$ \\
\hline 5 & $\begin{array}{l}\text { Hàm lượng nước } \\
\text { (mg/kg) }\end{array}$ & 98,2 & $\leq 200$ \\
\hline 6 & $\begin{array}{l}\text { Điện áp đánh thủng } \\
\text { tại khe hở 2,0 mm } \\
\text { (kV) }\end{array}$ & 45,1 & $\leq 35$ \\
\hline 7 & $\begin{array}{l}\text { Hệ số tổn hao điện } \\
\text { môi tại 25 }{ }^{\circ} \mathrm{C}(\%)\end{array}$ & 0,14 & $\leq 0,2$ \\
\hline 8 & $\begin{array}{l}\text { Hàm lượng lưu } \\
\text { huỳnh ăn mòn }\end{array}$ & Không có & Không có \\
\hline 9 & $\begin{array}{l}\text { Chỉ số axít (mg } \\
\text { KOH/g) }\end{array}$ & 0,009 & $\leq 0,06$ \\
\hline
\end{tabular}

\section{Kết luận}

Chất chống oxy hóa TBHQ đã cho thấy có hiệu quả trong việc cải thiện độ ổn định oxy hóa và làm chậm quá trình lão hóa hỗn hợp dầu cám và dầu hạt cải. Tuy nhiên trong điều kiện tiếp xúc với oxy và chịu tác động của nhiệt độ cao trong thời gian dài, hiệu quả của TBHQ không cao. Do đó, cần tiếp tục nghiên cứu hiệu quả của các chất chống oxy hóa khác. Hỗn hợp dầu cám và dầu hạt cải chứa TBHQ $(0,3 \mathrm{wt} \%)$ đã đạt tất cả các thông số theo qui định của tiêu chuẩn ASTM D6871 và có tiềm năng sử dụng làm dầu cách điện cho các máy biến áp cấp trung thế. 


\section{Lời cảm ơn}

Nghiên cứu này được tài trợ bởi Trường Đại học Cần Thơ.

\section{Tài liệu tham khảo}

[1] S. A. Ghani, N. A. Muhamad, Z. A. Noorden, H. Zainuddin, N. A. Bakar, M. A. Talib, Methods for Improving the Workability of Natural Ester Insulating Oils in Power Transformer Applications: A Review, Electric Power Systems Research, Vol. 163, 2018, pp. 655-667. https://doi.org/10.1016/j.epsr.2017.10.008

[2] M. Hrkac, P. Papageorgiou, I. Kosmoglou, G. Miatto, BIOTEMP $\AA$ Transformer Technology for Innovative Compact Substation, 7th Mediterranean Conference and Exhibition on Power Generation, Transmission, Distribution and Energy Conversion, Agia Napa, Cyprus, 2010. https://doi.org/10.1049/cp.2010.0954

[3] T. V. Oommen, Vegetable Oils for Liquid-Filled Transformers, IEEE Electr. Insul. Mag., Vol. 18, 2002, pp. 6-11.

https://doi.org/10.1109/57.981322

[4] D. V. Nguyen, L. P. Nguyen, T. N. Quach, An Investigation on Breakdown Properties of Paper Insulators Impregnated with Vegetable Oils for Transformers, IET Science, Measurement \& Technology, Vol. 13, 2019, pp. 1352-1361. https://doi.org/10.1049/iet-smt.2018.5580

[5] H. M. Wilhelm, M. B. C Stocco, L. Tulio, W. Uhren, S. G. Batista, Edible Natural Ester Oils as Potential Insulating Fluids, IEEE Trans. Dielectr. Electr. Insul., Vol. 20, 2013, pp. 13951401. https://doi.org/10.1109/TDEI.2013.6571461

[6] B. S. H. M. S. Y. Matharage, M. A. R. M. Fernando, E. Tuncer, M. A. A. P. Bandara, C. S. Kalpage, Coconut Oil as Transformer Liquid Insulation-Ageing and Simulated Thermal and Electrical Faults, Proceedings of the Annual Report Conference on Electrical Insulation and Dielectric Phenomena, Montreal, Canada, 2012. https://doi.org/10.1109/CEIDP.2012.6378911

[7] K. Varatharajana, D. S. Pushparanib, Screening of Antioxidant Additives for Biodiesel Fuels, Renewable and Sustainable Energy Reviews, Vol. 82, 2018, pp. 2017-2028. https://doi.org/10.1016/j.rser.2017.07.020

[8] H. M. Wilhelm, L. Feitosa, L. L. Silva, A. Cabrino, L. P. Ramos, Evaluation of in-Service Oxidative and Antioxidant Additive Consumption in Corn Oil Based Natural Ester Insulating Fluid, IEEE Trans. Dielectr. Electr. Insul, Vol. 22, 2015, pp. 864-869. https://doi.org/10.1109/TDEI.2015.7076786

[9] T. V. Oommen, C. C. Claiborne, E. J. Walsh, J. P. Baker, A New Vegetable Oil Based Transformer Fluid: Development and Verification, IEEE CEIDP, Canada, 2000.
https://doi.org/10.1109/CEIDP.2000.885288

[10] N. V. Dung, H. L. Huong, The Effect of Antioxidants on The Physical and Chemical Properties of Rice Oil, Corn Oil, Peanut Oil and Kraft Paper, IEEE Trans. Dielectr. Electr. Insul., Vol. 27, 2020, pp. 1698-1706. https://doi.org/10.1109/TDEI.2020.008422

[11] V. Mentlik, P. Trnka, J. Hornak, P. Totzauer, Development of a Biodegradable ElectroInsulating Liquid and its Subsequent Modification by Nanoparticles, Energies, Vol. 11,2018 , pp. 508-524. https://doi.org/10.3390/en11030508

[12] N. V. Dung, Application of Vegetable Oil-Based Insulating Fluids for Transformers: A Review, VNU Journal of Science: Natural Sciences and Technology, Vol. 35, 2019, pp. 1-15, https://doi.org/10.25073/2588-140/vnunst.4936. (in Vietnamese)

[13] N. Beltrán, E. Palacios, G. Blass, Potential of Jatropha Curcas Oil as a Dielectric Fluid for Power Transformers, IEEE Electr. Insul. Mag, Vol. 33, 2017, pp. 8-15. https://doi.org/10.1109/MEI.2017.7866674

[14] K. Bandara, C. Ekanayake, T. Saha, H. Ma, Performance of Natural Ester as a Transformer Oil in Moisture-Rich Environments, Energies, Vol. 9, 2016, pp. 1-13. https://doi.org/10.3390/en9040258

[15] A. Raymon, P. S. Pakianathan, M. P. E. Rajamani, R. Karthik, Enhancing the Critical Characteristics of Natural Esters with Antioxidants for Power Transformer Application, IEEE Trans. Dielectr. Electr. Insul., Vol. 20, 2013, pp. 899-912. https://doi.org/10.1109/TDEI.2013.6518959

[16] S. S. Kumar, M. W. Iruthayarajan, M. Bakrutheen, S. G. Kannan, Effect of Antioxidants on Critical Properties of Natural Esters for Liquid Insulations, IEEE Trans. Dielectr. Electr. Insul., Vol. 23, 2016, pp. 20682078. https://doi.org/10.1109/TDEI.2016.7556480

[17] A. A. Zaky, I. Y. Megahed, C. Evangelou, The Effect of Organic Additives on the Breakdown and Gassing Properties of Mineral Oils, J. Phys. D Appl. Phys., Vol. 9, 1976, pp. 841-849. https://doi.org/10.1088/0022-3727/9/5/017

[18] M. Unge, S. Singha, N. V. Dung, D. Linhjell, S. Ingebrigtsen, L. E. Lundgaard, Enhancements in the Lightning Impulse Breakdown Characteristics of Natural Ester Dielectric Liquids, Applied Physics Letters, vol. 102, 2013, pp. 1-4. https://doi.org/10.1063/1.4803710

[19] S. Tenbohlen, M. Koch, Ageing Performance and Moisture Solubility of Vegetable Oils for Power Transformers, IEEE Trans. Power. Del., Vol. 25, 2010, pp. 825-830.

https://doi.org/10.1109/TPWRD.2009.2034747 